\title{
Spectrum Efficiency Enhancement in Dynamic Space Coded Multiple Access (DSCMA) System
}

\begin{abstract}
In cellular mobile communication systems using coded modulations, the spectrum efficiency of the system is related to the number of available codes. Recently, large area synchronous (LAS) CDMA codes, which exhibit a region called interference free window (IFW) within some delay-spread, have been accepted as one of fourth-generation (4G) wireless communication systems. However, the number of synthesized LAS codes is very low due to the low duty ratio of the sequence. In this paper, a dynamic space coded multiple access (DSCMA) scheme which utilizes the spatial diversity from smart antenna system is proposed to overcome the low spectrum efficiency in LAS CDMA system. In the DSCMA a modified version of LAS codes called LAS even ternary (LAS-ET) codes is proposed. These codes are used together with a novel algorithm called dynamic space code (DSC), which will decrease the code length so that the spectrum efficiency can be increased. By taking advantage of dynamic code reuse assignment in spatial diversity, the spectrum efficiency of the DSCMA can be further increased significantly.
\end{abstract}

Keyword: Spectrum efficiency, LAS CDMA, IFW, Smart antenna, Code reuse, Duty ratio, Beamwidth 